\title{
Return Channel for the Brazilian Digital Television System-Terrestrial
}

\author{
Luís Geraldo P. Meloni ${ }^{1}$ \\ ${ }^{1}$ Department of Communications \\ School of Electrical and Computing Engineering \\ State University of Campinas \\ P.O.Box 6101, Zip 13083-852 - Campinas - SP - BRAZIL \\ meloni@decom.fee.unicamp.br
}

\begin{abstract}
Recently, it has been proposed the use of WiMAX for the return channel in digital television systems using a new frequency profile bellow $1 \mathrm{GHz}$, which has been called WiMAX-700. The profile operates from $400 \mathrm{MHz}$ to $960 \mathrm{MHz}$ as primary band, which includes the UHF band, and optionally from $54 \mathrm{MHz}$ to $400 \mathrm{MHz}$ as secondary band. This work presents some aspects of the WiMAX700 technology and some simulation results of models designed to determine the system capacity concerning the number of actives subscriber stations, according to specific proposed scenarios, traffic sources profiles, specifics propagation, and coverage conditions. The results presented in this work provide elements to determine the suitability of WiMAX-700 technology as the return channel for the interactive digital television applications.
\end{abstract}

Keywords: WiMAX-700, IEEE 802.16, 700MHz profile, Digital Television Systems, Return Channel, NS2

\section{INTRODUCTION}

Brazil has recently defined its Digital TV System based on the Japanese ISDB-T. The Brazilian system (SBTVD-T) uses the same modulation technique of the ISDB-T system, also known as BST-OFDM - Band Segmented Transmission - Orthogonal Frequency Division Multiplexing, which consists of a series of frequency blocs called OFDM segments that provide DTV transmission for fixed and mobile receivers simultaneously. The OFDM segments use different transmission parameters allowing a hierarchical transmission.
Among the Brazilian innovations, there is a new proposal of middleware that is based on the NCL language, that is a declarative language for hypermedia documents authoring and object synchronizing. Another important innovation regards a new WiMAX profile that cover the UHF and VHF television bands which is the main purpose of this paper.

The SBTVD-T standards are based on ARIB standards. Concerning the Return Channel (RC), among the technologies currently previewed in ARIB are dialup modems, ISDN for wired lines and PDC - Personal Digital Cellular and PHS - Personal Handy-Phone System for wireless. PDC is a standard developed and used exclusively in Japan. The SBTVD-T has complemented the above return channel technologies by including WiMAX/WiMAX-700, GSM/GPRS, and CDMA2000/1xRTT.

The main motivation to include wireless standards as return channel in the SBTVD-T is the increasing observed rate of mobile phone users in Brazil, having surpassed the number of fixed lines by more than two folds. It is expected that the same rising rate will be observed in wireless Internet access in this decade.

The WiMAX-700 is a new WiMAX profile that covers $400 \mathrm{MHz}$ to $960 \mathrm{MHz}$ band, including all UHF TV channels and also optionally including the VHF band, by including a secondary band from $54 \mathrm{MHz}$ to $400 \mathrm{MHz}$. The WiMAX-700 presents several advantages over current profiles: better indoor penetration, higher propagation reach of up to $65 \mathrm{~km}$, and lower cost due to the fact that the system uses components and modules of the entertainment electronics that present lower cost over the 
telecommunications and information technologies. The higher propagation reaches are important in cities with low density population allowing the use of only one base station in the return channel network.

This paper is structured as follow: section II deals with WiMAX-700 as return channel for the SBTVD-T, section III presents WiMAX-700 channeling techniques, section IV presents some results of a simulation environment including several traffics estimates; and finally section $\mathrm{V}$ concludes the paper.

\section{WIMAX AS RC FOR TEH SBTVD-T}

The initial idea of interactivity in Digital Television is the possibility of data communications between receiver stations with applications and services eventually available in the broadcaster's signal. Data communications to the majority amount of set-top boxes is implemented by means of data carrousel of the broadcaster transmitter station. The inverse link data communications normally uses a subsystem called return channel, which allows in general in the DTV systems only half duplex communications and allows low bit rates transmissions, such as the Return Channel Terrestrial from DVB-T. In the SBTVD$\mathrm{T}$ the return channel is full duplex and the middleware explores this functionality.

One of the available technologies for interactivity channel is WiMAX which offer great communication capability for the DTV return channel subsystem. The Figure 1 shows the return channel scheme supported by the SBTVD-T.

The figure shows the three subsystems of the DTV. The broadcast subsystem generates the transport stream for broadcasting transmission. The transport stream multiplexes video, audio, and data. The video coding of the SBTVD-T uses the H.264, level 4.0, offering a high resolution of 1080i. Audio coding uses the HE-AAC standard. The receiver implements the inverse operation for disposing data for users. Finally, the return channel allows the communications between uses and interactive applications at the broadcaster or at any server connected to the Internet, outside the broadcast facilities. In this scheme the access to Internet is independent of the return channel technology.

WiMAX has the advantage to full duplex transmission, offering high bitrate to users, besides the compatibility with packets networks also offering access to Internet.

\subsection{WIMAX-700 APPLICATION IN THE SBTVD-T}

The use of WiMAX at the $700 \mathrm{MHz}$ profile offers several advantages [1] [2]: better indoor penetration when compared to other profiles; better signal reach of up to
$65 \mathrm{~km}$, offering return channel to low density population areas with only one base station, and also due to the fact of using entertainment components, the final system costs are lower when compared to the communications and information technologies.

These advantages justify the interest of defining a new frequency profile of WiMAX-700 which includes the UHF band and optionally the VHF band. The Figure 2 illustrates wireless transmissions of the proposed return channel for the SBTVD-T.

The WiMAX-700 radiofrequency solution uses a wireless modem for the previewed frequency band. The modem communicates with the base station offering Internet access. The modem disposes the return channel to the set-top box by means of the USB or Ethernet interfaces. The modem may also include a firewall or router functionalities for Internet access to other devices such as PCs, PDAs or others. The WiMAX modem can also be integrated inside the STB.

Several important aspects concerning the Figure 2 scheme are relevant. The return channel is not necessarily linked to the broadcast station, i.e., the broadcast station and the return channel provider may be physically separated. A common scenario in a big city would be for instance the use of a single broadcast antenna in an appropriated high location and several base stations spread over the city in a distribution similar to the cells as in mobile services. In his manner, the system deployment depends on the existence of a entity denominated Return Channel Provider. This entity may be private or public, and it has fundamental importance, it is responsible for planning services, system deployment, users' registry, and base station operations, among other responsibilities.

Some WiMAX-700 requirements are presented in Table 1 for the SBTVD-T.

\subsection{Network ArChitecture}

The proposed return channel provides a wireless communications to a nearby base station. This scheme allows the coexistence of DTV broadcast networks with a wireless telecommunications networks. The networks architecture is shown in Figure 3. The base station indicated by letter A is responsible by TV signal broadcast covering the service area. The return channel is implemented by means of a transceiver (sender and receiver) at the user set-top box, which besides the digital TV reception; it has a low-power transceiver that allows data communications to a nearby return node representing a base station. Letter $B$ in Figure 3 represents users and letter $C$ the return nodes. This scheme is similar to mobile telephony where the coverage cells are defined by the radio bases. It is also possible to use the same conventional antenna for VHF and UHF for WiMAX-700 targeting low cost receivers.

In this scheme the return channel nodes have equip- 


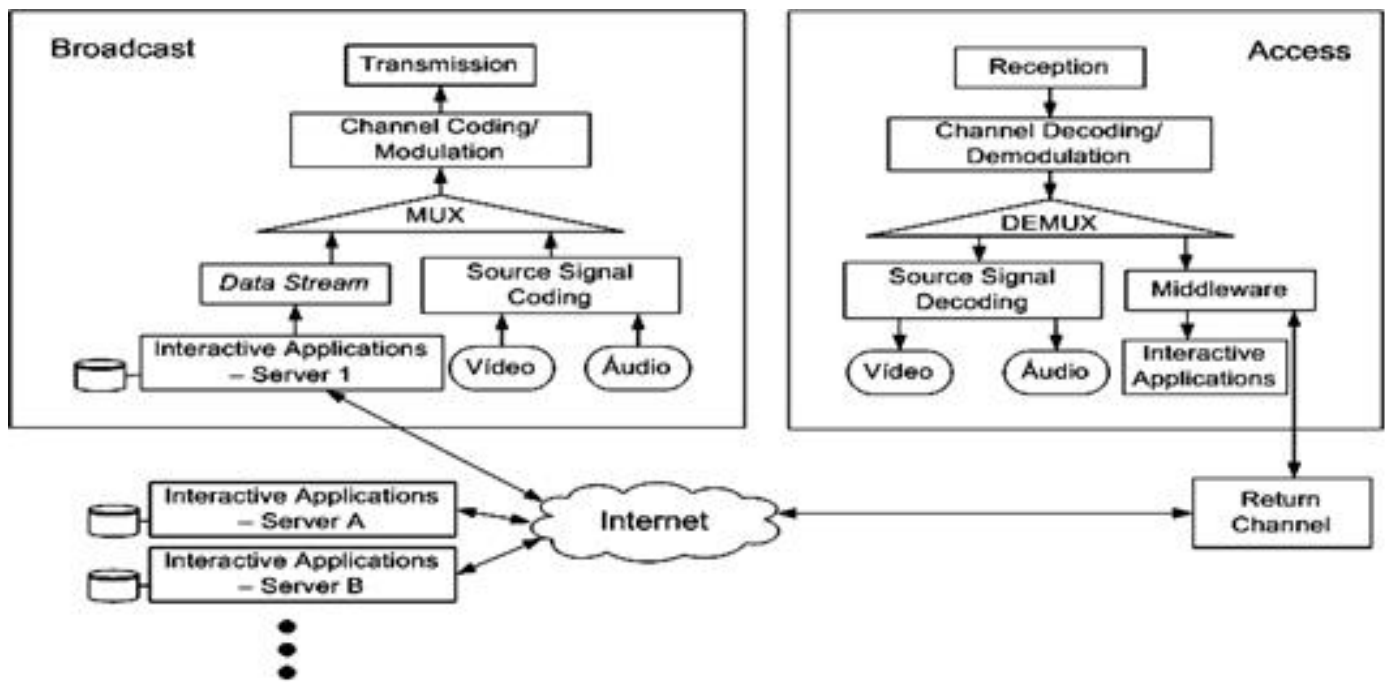

Figure 1. Full-duplex Return Channel with Internet Access.

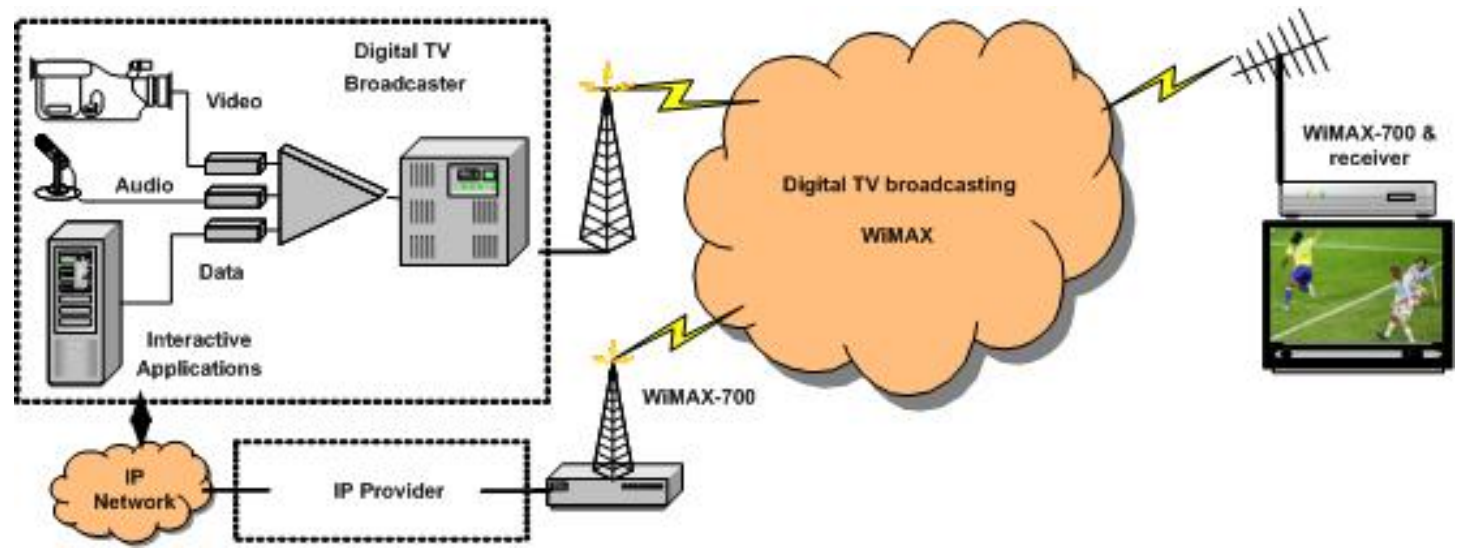

Figure 2. Return Channel Architecture with WiMAX-700.

ments for wideband Internet access, representing by letter $\mathrm{D}$ in Figure 3. The responsability of these connections are from the return channel provider, and it can be provided by different forms. In some places, depending on the availability, optical linking, Ethernet or other can be used; another alternative of lower cost is the use of pointto-point wireless links.

The STB may also use different return channel modality, the SBTVD-T middleware manages which return channel will be in use.

The IEEE802.16 standard defines a specification for air interface for wireless Metropolitan Area Networks (MAN). This wireless MAN may offer wideband Internet access, an alternative for other access modalities such as cable or fiber optics, offering a less expensive solution. It is an access solution for the last mile that fulfills the requirements of the return channel of the SBTVD-T. The WiMAX-700 is included in the SBTVD-T specifications, which details the necessary enhancements for operating witch the DTV system in the new frequency profile.

The use of IEEE802.16 as return channel for DTV offers several advantages. Upgrades and enhancements of the WiMAX which occurs along the standard evolution may be promptly incorporated in the return channel, such as the recent IEEE802.16e revision that allows mobility. With this revision is possible to use WiMAX in mobile DTV terminals, considering naturally the respective upgrade in the SBTVD-T. 
Table 1. Some Characteristics of IEEE802.16 Standard.

\begin{tabular}{l|l}
\hline Functional Requirements & Description \\
\hline Physical Layer (PHY) & - According to the IEEE802.16 Standard in [3] and [4]; \\
& - Coverage radius may reach approximately 65 km, appropriated to rural areas; \\
& - NLOS - Non Line of Sight; \\
& - Operation in the 400 MHz to 960 MHz, and optionally in the VHF band. \\
\hline Medium Access Layer (MAC) & - According to the IEEE802.16 Standard in [3] and [4]; \\
& - Compatible with system architectures base on packets, such as TCP-IP, IP proto- \\
& cols, Ethernet/IEEE 802.3, etc. \\
& - Connection oriented services; \\
& - PMP - Point to Multipoint; \\
& - Manageable QoS. \\
\hline Interface with Other Modules & - USB preferably; \\
& - Ethernet IEEE 802.3; \\
& - Compatibility with upper layer network and transport protocols. \\
\hline Mobility & - Allows receiver mobility - IEEE802.16e-2005. \\
\hline Identification & - Each modem has a single identifier (ID). \\
\hline
\end{tabular}

\section{WIMAX-700 CHANNELING}

Several improvements have been introduced in the WiMAX-700 mainly concerning the synchronization and equalization techniques, [5], [6], and [7]. This section review the scheme proposed in the SBTVD-T concerning spectrum channeling.

WiMAX-700 allows wireless communications in the frequency of $400 \mathrm{MHz}$ to $960 \mathrm{MHz}$, which includes the UHF (Ultra High Frequency) band, and optionally includes the VHF (Very High Frequency) band.

The channeling method allows simultaneously operation of the digital transmission systems by means of OFDM/OFDMA techniques or other techniques in such way to allow coherent operation with the analogue or digital television systems. The method allows the reutilization of frequencies for transmission using OFDM/OFDMA modulation in free channels at the defined frequency profile, operating harmonically with other systems, with emphasis to the television broadcasting and mobile telephone services.

The television systems around the world use the following bandwidths: $6 \mathrm{MHz}, 7 \mathrm{MHz}$ or $8 \mathrm{MHz}$. With purpose to harmonize WiMAX with the several standards, the following bandwidths and the respective masks have been defined to the WiMAX-700:

- $1.5 \mathrm{MHz}, 2 \mathrm{MHz}, 3 \mathrm{MHz}, 6 \mathrm{MHz}$ or $12 \mathrm{MHz}$ - for television system with $6 \mathrm{MHz}$ channeling,

- $1.75 \mathrm{MHz}, 3.5 \mathrm{MHz}, 7 \mathrm{MHz}$ or $14 \mathrm{MHz}$ - for television system with $7 \mathrm{MHz}$ channeling,

- $2 \mathrm{MHz}, 4 \mathrm{MHz}, 8 \mathrm{MHz}$ or $16 \mathrm{MHz}$ - for television system with $8 \mathrm{MHz}$ channeling.
In WiMAX-700 the following channeling is defined by the initial frequency:

$$
f_{i}=S_{f}+n B T+k B W
$$

where: $S_{f}$ is the starting frequency of the TV systems in $\mathrm{MHz}$, according to different global systems, $n$ e $k$ are integers,

$$
B T \in\{6,7,8\}[M H z]
$$

represents television channel bandwidth; and

$$
B W \in\{1.5,1.75,2,3,3.5,4,6,7,8,12,14,16\}[M H z]
$$

defines the bandwidths of WiMAX channels. For instance, for operation of WiMAX-700 in Brazil, the following values are used:

$$
\begin{gathered}
S_{f} \in\{54,76,174,470\}[M H z], \text { and } \\
B W \in\{1.5,2,3,6,12\}[M H z] .
\end{gathered}
$$

Generally the WiMAX systems employ frequency reuse factor of 3, or even 1, in which case is implemented an internal frequency reuse, by means of a sub-channeling or frequency hopping. Such reuse factors of 3 or 1 may be difficultly considered in the $600 \mathrm{MHz}$ or $700 \mathrm{MHz}$ bands. In this case, the signal propagation has different characteristics, and the reuse patterns may be similar to those of mobile phones operating at frequencies bellow $1 \mathrm{GHz}$.

Several reuse patterns are possible; some of them are illustrated in Figure 4 and Figure 5. Figure 4 shows reuse pattern of $4 \times 1$ and $4 \times 3$, for only one sector cells or three sectors of $120^{\circ} \mathrm{C}$. In these cases, 4 and 12 frequencies are used respectively. The base station antennas are placed in the center of each hexagon. In the first case, using 1.5 


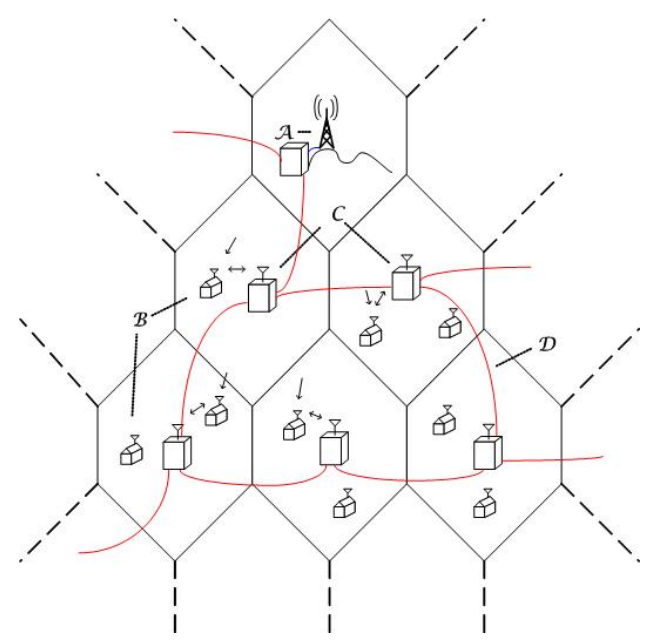

Figure 3. WiMAX-700 Architecture as DTV Return Channel.

$\mathrm{MHz}$ WiMAX it is necessary one $6 \mathrm{MHz} \mathrm{TV}$ channel, and in the second case, using $2 \mathrm{MHz}$ WiMAX, it is necessary to use four $6 \mathrm{MHz}$ TV channels. Figure 5 shows reuse pattern of $7 \times 1$ and $7 \times 3$, for only one sector cells or three sectors of $120^{\circ} \mathrm{C}$. In these cases 7 and 21 frequencies are used respectively. In the first case, using 1.5 MHz WiMAX it is necessary two $6 \mathrm{MHz}$ TV channel, and in the second case, using $2 \mathrm{MHz}$ WiMAX, it is necessary to use seven $6 \mathrm{MHz}$ TV channels. Naturally, other reuse frequency patterns are possible within WiMAX-700 systems.

\section{WiMAX-700 Capacity Simula- TION}

For purposes of performance analysis, several simulation models of the IEEE 802.16 wireless network have been implemented to simulate air interface traffic of the return channel of DTV [2]. The model reproduces the mechanism of IP packets transmission between a base station and the subscriber stations (SS) of a sector controlled by the base station (BS).

The simulator uses the NS-2 environment [8], which has a set of elements to simulate data transmission networks, for example IP protocol, wire and wireless transmissions systems, and MAC protocols (IEEE 802.11 and TDMA). A new element was developed to simulate the IEEE 802.16 protocol which was employed over several rounds of simulations to analyze the IEEE 802.16 network capability performance for DTV return channel. The NS-2 is a discrete events simulator specialized in computers networks simulation. The NS-2 is open source a software and an object-oriented simulator, written in $\mathrm{C}++$, which allows code reutilization and modular- ity. These characteristics make possible a fast model development of the MAC layer of the IEEE 802.16 protocol [9], [10], and [11].

\subsection{Scenarios of Simulation}

The simulation analysis uses only a basic scenario which reproduces operation in a sector controlled by one base station. The scenario was simulated several times, using distinct parameters of propagation, distinct parameters of SS distributions, different types of SS traffic as well as the number of SS.

In general, two points of view are very important for mobile systems: signal propagation and channel traffic capacity. The signal propagation determines the major radius of cells (or sector), so that the received power intensity is appropriate for a good system performance. The traffic capacity determines the major number of SS supported by a cell (or sector). In general, in the urban areas, the cells radius is limited by the traffic capacity and in the rural areas by the propagation features. The main purpose of the simulation model is to allow an analysis concerning the traffic capacity point of view.

It is specified the transmission mode of each node representing a base station in the model. The transmission mode determines the modulation scheme (BPSK, QPSK, 16-QAM and 64-QAM - for IEEE802.16d) and the error correction code scheme, both defined by the standard. These parameters determine the transmission rate, as well as the error rate for a specific signal to noise ratio level. In a real network, the transmission modes are determined from the power level, interference and error rate measurements of the received signal. These parameters are entries for the simulation model.

In order to specify the sectors traffic capacity, two scenarios were used for wireless propagation and SSs distributions. Without loss of generality, both models have a circular sector of one $\mathrm{km}$ radius. From the traffic point of view, the cell radius and the sectors geometries are important only to determine the number of SSs which utilize each transmission mode. For example, a circular sector (360 degrees) that covers a certain area with a certain SS density has the same number of SS of such a sector of 120 degrees covering an area three times bigger. From the simulation view point, the capacity in both cases is the same. The factor that introduces any cell capacity variation is the signal propagation. The number of SS distribution operating in each operation mode is slightly different in function of signal attenuation due to path losses. The COST 231 [12] propagation model was used to analyze the cells capacity and two approaches were defined depending on loss factors. The first approach, called P26, has considered a loss factor of 2.6, which is a typical factor for urban and suburban areas. The second, called P35, has considered a 3.5 loss factor, which represents an area 


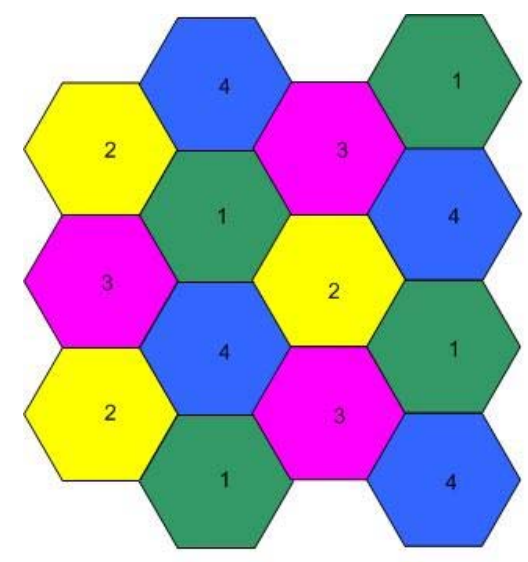

$4 x_{1}$ Pattern

Four $1.5 \mathcal{M H z}$ channels One $\mathcal{T} \mathcal{V}$ channel

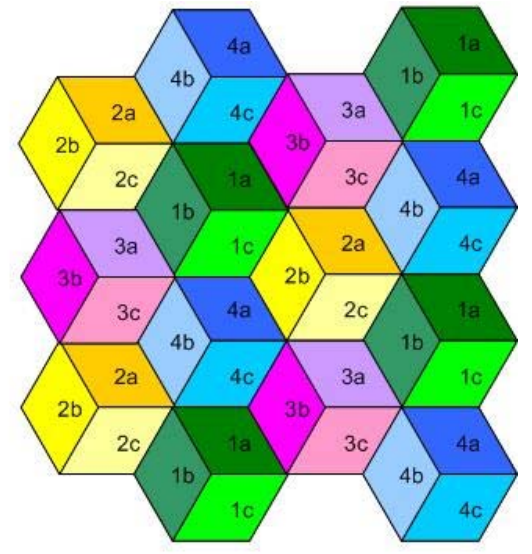

$4 \times 3$ Pattern

Twelve 2.o $\mathcal{M H}$ H channels Four $\mathcal{T V}$ channels

Figure 4. Reuse Frequency Patterns.

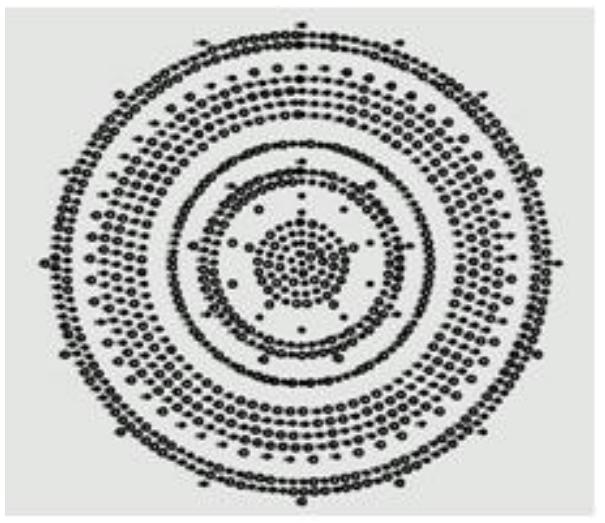

Figure 6. SS Distribution for P26 Scenario.

with bad propagation characteristics, such as a density urbanized areas with irregular topography.

In order to define the number of SS operating in each transmission mode, it was considered an homogeneous distribution of SS in a circular area.

Six circulars sectors were created in both scenarios calculated from the reach of the transmission signal in each mode. A percentage SS distribution was defined for each sector. This percentage is equal the rate between the sector area and the total area of the cell. The total number of SS in the cell is defined for each simulated scenario. The simulation model distributes the SS in each sector and associates them to the corresponding transmission mode (Table 2).

The transmission mode in the direct and reverse path is different for a same sector. The difference between approaches P26 (Figure 6) and P35 (Figure 7) is that the approach P35 has a higher SS concentration in the higher transmission rates. In conditions of bad transmissions, a higher transmission power is needed (or in an equivalent way, a reduction of the cell radius). An effect also related to higher signal attenuation in P35 scenario, is the small sectors area corresponding to a particular modulation scheme at the cell border, as compared to P26 scenario which has a smaller attenuation factor. Consequently, the approach with worst propagation factor is paradoxally the scenario more favorable regarding traffic flow.

Four traffic profiles were specified in simulations: WEB, low rate bidirectional stream, e-mail and low rate client/server session communications. These also define five different profiles distributions which were considered for the rounds of simulation (Table 3). The table presents the distributions of the active SS that generate the respective traffic according to the percentage shown in the table.

\begin{tabular}{c|c|c|c}
\multicolumn{3}{c}{ Table 2. Percent Distribution of SS in the Sectors. } \\
\hline \multicolumn{2}{c}{ Mode } & \multicolumn{2}{c}{ SS } \\
\hline UL & DL & P26 & P35 \\
\hline QPSK 1/2 & QPSK 3/4 & 30 & 23 \\
\hline QPSK 3/4 & 16-QAM 1/2 & 41 & 37 \\
\hline 16-QAM 1/2 & 16-QAM 3/4 & 9 & 9 \\
\hline 16-QAM 3/4 & 64-QAM 2/3 & 12 & 15 \\
\hline 64-QAM 2/3 & 64-QAM 3/4 & 1 & 2 \\
\hline 64-QAM 3/4 & 64-QAM 3/4 & 7 & 14 \\
\hline
\end{tabular}




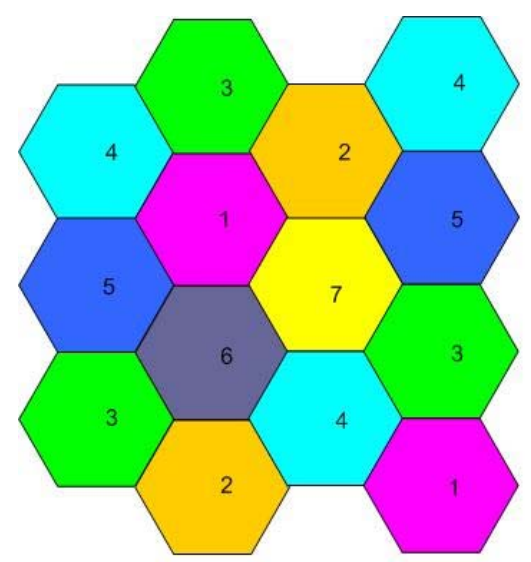

$7 \times 1$ Pattern

Seven $1.5 \mathcal{M H} \mathcal{H}$ channels

Two $\mathcal{T} \mathcal{V}$ channel

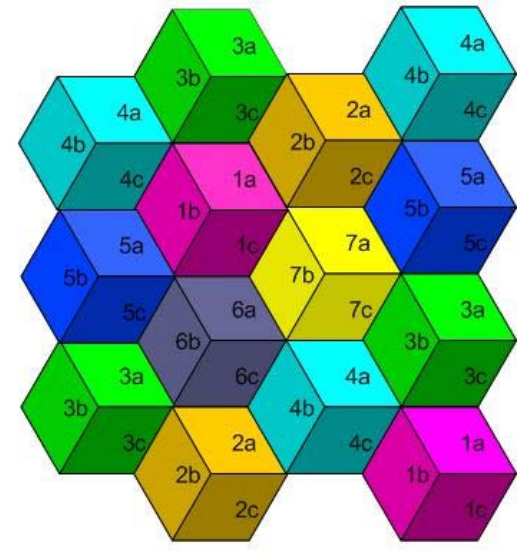

$7 x_{3}$ Pattern

Twenty One 2.o $\mathcal{M H z}$ channels Seven $\mathcal{T} \mathcal{V}$ channels

Figure 5. Reuse Frequency Patterns.

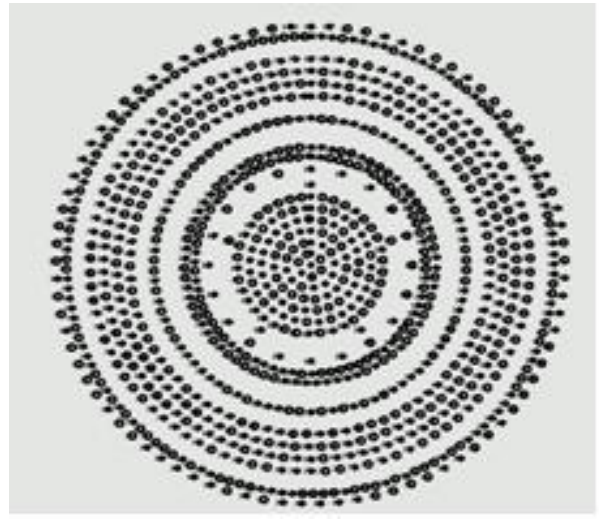

Figure 7. SS Distribution for P35 Scenario.

Next, the characteristics of each traffic profile are presented:

1. Web: This profile utilizes the connections source model using default parameters of the Packmime model (PackMimeHTTP - Bell Labs) [13] with rate of 1 page access per 2 minutes per station.

2. Low Rate client/server Session: to simulate this profile a new agent was created in the NS-2 with the following parameters:

- Request packets length: exponential distribution with mean of 512 bytes.

- Response packets length: exponential distribution with mean of 50 Kbytes.

- Connections rate done by user: 10 per hour.
- Request packets number per connection: exponential distribution with mean of 8 .

- Time delay to send response packets after reception of request packets: 0 .

- Time delay to send the next request packets after reception of response packets: negative exponential distribution with mean of 20 seconds.

3. E-mail: the profile utilizes the On-Off source model (Application/Traffic/Exponential) with the following parameters:

Receiving E-mails:

- Transmission rate in the active state: 240 kbits/s

- Messages length: 15 kbytes.

- Activity period mean time: 4 seconds.

- Inactivity period mean time: 1200 seconds.

Sending E-mails:

- Transmission rate in the active state: $16 \mathrm{kbits} / \mathrm{s}$.

- Messages length: 200 bytes.

- Activity interval mean time: 0.1 second.

- Inactivity interval mean time: 600 seconds.

4. Low Rate Bidirectional Stream: This profile uses the model of simple variable rate (Application/Telnet), with the parameters:

- Transmission rate: 1 message each 10 seconds.

- Message Length: 100 Bytes. 
The simulation used the NS-2 routing protocol DSDV (Destination Sequence Distance Vector) [8]. This protocol allows systems simulation in the radio environment. In the model, the IP layer of each SS has an agent who sends periodically packets of routing to the neighbors SS. When the agents receive the packets, they obtain the necessary information to construct the routing table, which will be used to redirect the traffic. All the rounds of simulation have both the application traffic and that traffic in the IP layer. It is worth to remark that the DSDV protocol has been used for the simulation purposes, but may not be present in WiMAX environment. Several rounds of simulation were executed for each arrangement of propagation scenario and for each traffic scenario, as varying the number of SS in the sector. The number of SS was assumed to vary between 500 and 1000 , with increments of $50 \mathrm{SS}$ for each simulation step.

Table 3. Percent Distribution of SS in the Sectors.

\begin{tabular}{c|c|c|c|l}
\hline Profile & WEB & Session & E-mail & $\begin{array}{l}\text { Bidirectional } \\
\text { Stream }\end{array}$ \\
\hline CI-Low & $0 \%$ & $0 \%$ & $0 \%$ & $100 \%$ \\
\hline CI-1 & $0 \%$ & $0 \%$ & $90 \%$ & $10 \%$ \\
\hline CI-2 & $0 \%$ & $50 \%$ & $45 \%$ & $5 \%$ \\
\hline WEB-1 & $25 \%$ & $37 \%$ & $34 \%$ & $4 \%$ \\
\hline WEB-2 & $50 \%$ & $25 \%$ & $22 \%$ & $3 \%$ \\
\hline
\end{tabular}

\subsection{Configuration Model}

A series of simulations were done using a traffic source of constant rate. The traffic volume produced by traffic sources was greater than system capacity, allowing analyzes of the MAC layer at maximum dataflow. In this way, the simulated system behavior is deterministic, and the comparison between simulation results and calculations analyzes is simple. Table 4 and Table 5 present these results. They present MAC and PHY layers (including protocol IEEE 802.16 overhead), dataflow (bytes per second). MAC layer dataflow amount had been compared with transmission nominal rate (operation using $3.5 \mathrm{MHz}$ bandwidth). The reverse path had a gain of about $80 \%$ and direct path of about $74 \%$. These values are due to protocol packaging, which needs additional bits transmission to control the preamble transmission times, guard time, information transmission about system management and allocation band mechanism used for simulation.

\subsection{Simulation Models}

The following simulation models were developed.

1. TDD Model: A MAC layer model has been developed according to the IEEE 802.16 system operating in the OFDM/TDD mode. The model represents
Table 4. Model Validation Results - Reverse Path (Dataflow in bytes/s, Nominal Rate and Efficiency)

\begin{tabular}{c|c|c|c|c}
\hline \multicolumn{5}{c}{ Reverse Path } \\
\hline Mode & PHY & MAC & Rate & Efficiency \\
\hline BPSK 1/2 & 151,200 & 140,800 & 1.47 & $77 \%$ \\
\hline QPSK 1/2 & 302,400 & 291,200 & 2.94 & $79 \%$ \\
\hline QPSK 3/4 & 453,600 & 441,600 & 4.41 & $80 \%$ \\
\hline 16-QAM 1/2 & 604,800 & 592,800 & 5.88 & $81 \%$ \\
\hline 16-QAM 3/4 & 907,200 & 893,600 & 8.82 & $81 \%$ \\
\hline 64-QAM 2/3 & $1,209,600$ & $1,181,700$ & 11.76 & $80 \%$ \\
\hline 64-QAM 3/4 & $1,360,800$ & $1,331,350$ & 13.24 & $80 \%$ \\
\hline
\end{tabular}

Table 5. Model Validation Results - Direct Path (Dataflow in bytes/s, Nominal Rate and Efficiency)

\begin{tabular}{c|c|c|c|c}
\hline \multicolumn{5}{c}{ Direct Path } \\
\hline Mode & PHY & MAC & Rate & Efficiency \\
\hline BPSK 1/2 & 136,800 & 136,200 & 1.47 & $74 \%$ \\
\hline QPSK 1/2 & 273,600 & 273,000 & 2.94 & $74 \%$ \\
\hline QPSK 3/4 & 410,400 & 409,800 & 4.41 & $74 \%$ \\
\hline 16-QAM 1/2 & 547,200 & 546,600 & 5.88 & $74 \%$ \\
\hline 16-QAM 3/4 & 820,800 & 820,200 & 8.82 & $74 \%$ \\
\hline 64-QAM 2/3 & $1,094,400$ & $1,093,800$ & 11.76 & $74 \%$ \\
\hline 64-QAM 3/4 & $1,231,200$ & $1,230,600$ & 13.24 & $74 \%$ \\
\hline
\end{tabular}

mainly the most essential protocol elements necessary to obtain dataflow and delay times, which are: mechanism for band allocation in direct and reverse path, mechanism for band request from SS to base station, adaptive modulation and data packaging in the MAC and PHY layers. We have looked to represent these elements according to the IEEE 802.16 specification. The band allocation mechanism is not included in this specification. So, a simple system is modeled, using the Round Robin type, which allocate an equal opportunity period for each SS for transmission. The opportunity period is an entry parameter of the model (quota). As the SS use different bit rates, the mechanism allocates higher band to the SS with major bit rates. The developed model is quite flexible and allows configuring several parameters. Next, a list of parameters and used values in simulations are presented. These values are related to IEEE 802.16 system using 3.5 MHz channel band, frames of $20 \mathrm{~ms}$ in TDD transmission mode. There are 16 sub-channels in reverse path. The model parameters are:

- Backoff window minimum length (2).

- Backoff window maximum length (16). 
- MAC layer packets overhead (12 octets).

- Frame length (20 ms).

- Symbol duration (68 $\mu \mathrm{s})$.

- Frame guard time $(300 \mu \mathrm{s})$.

- Number of direct path sub-channels (1).

- Number of reverse path sub-channels (16).

- Number of bytes per symbol per transmission mode $(12,24,36,48,72,96,108)$.

- Multiplexing type (TDD)

- Quota per station used in band allocation algorithm (direct path: 4 slots, reverse path: 64 slots).

- Sub-frame minimum duration (number of slots) of reverse path in TDD mode (8).

- Burst minimum length (number of slots) to each transmission mode (direct path: 1 to all modules; reverse path: $8,4,4,2,2,1,1)$.

- Number of channels for band allocation request (48)

- Number of codes for band allocation request (8).

- Number of opportunities for band allocation request per frame (2).

- Ranging phase duration (number of symbols) in reverse path (3).

- First burst (preamble) duration (number of slots) in direct path (5).

- Periodicity of statistical logging (1 second).

- Trace level (1).

The level trace 1 generates a registry file to store and collect statistical. These include packets total dataflow (number of octets) and average time delay in direct and reverse paths. Dataflow is measured in enlace layer (MAC entry), which regards only the traffic in upper layers, and in Physical layer (MAC exit), which considers the additional bytes in packaging of the MAC layer. The packets delay time considers the waiting time of transmission and its own transmission time.

2. FDM Model: The FDM model was developed, but not used in the Digital TV return channel study, due to the fact that it presents disadvantages in flexibility, once the available band for direct and reverse paths is constant, in contrast to TDD mode where band share is dynamic.

\subsection{Results Analyzes}

The tables 6, 7, and 8 present results for the same traffic and propagation scenarios for different number of SS. They present dataflow in the physical layer, including IEEE 802.16 protocol overhead, and dataflow in the enlace layer (IP dataflow). All rounds simulate 10 minutes of network operation. To analyze results, only the final 2 minutes have been considered to ensure that the data output were in a statistical stable region. The obtained results correspond to confidence interval lower then $1 \%$ average values to a confidence level of $95 \%$. In the result analyzes all rounds of simulation whose packets time delay were stationary around its mean value were considered. This is the reason why the maximum number of SS is different to each traffic and propagation scenario. An important point to observe in the results is the traffic impact produced by the routing DSDV mechanism. DSDV agents investigate the neighbors, sending a broadcast message to the radio interface. When agents receive the message, they send a response informing their addresses. This traffic increases with the square of SS number. In the simulated approaches, that traffic was significant and it allowed evaluate small dataflow traffic effect originated by broadcast messages.

The maximum and minimum total averages dataflow (reverse + direct links) achieved in simulations were 567 and $292 \mathrm{kbytes} / \mathrm{s}$ (IP layer). The IEEE 802.16 protocol is optimized to broadband traffic. DSDV traffic and others simulated traffics applications were small data bursts. The maximum and minimum rate difference shows that system can be used in a scenario of great number of actives SS transmitting with small rates simultaneously, but also shows that it can has a great variation in efficiency in function of traffic profile. It is also important to observe that the behavior of dataflow is not monotonous with the number of subscriber stations. The mainly reason for that is the distinct spatial traffic sources distribution for each scenario. Because relatively small number of SS transmitting with great rates (vide Table 2), a distribution variation can cause a significant effect in the results.

\subsection{RF SPECTRUM USE}

Using the spectral efficiency reported on technical literature, Table 9 shows the number of TV channels to be used considering the use of WiMAX-700 as return channel. In this table, it has been considered 4 cells with 3 sectors each one. It has also been considered the following bandwidths $1.5,1.75,2,3,3.5,4$ e $5.5 \mathrm{MHz}$. The table shows the mean bitrate per user operating simultaneously in the system (uplink and downlink). It has been considered a user density of 500 users per sector and a spectral efficiency of $2 \mathrm{bps} / \mathrm{Hz}$ (a conservative result). For a bandwidth of 3.0 MHz, it would be necessary $6 \mathrm{TV}$ channels, which would offer a bitrate of $12 \mathrm{kbps}$. It is im- 
Table 6. Results of CI-2/P26 Scenario)

\begin{tabular}{c|c|c|c|c|c|c}
\hline \multicolumn{7}{c}{ CI-2/P26 } \\
\hline SS & \multicolumn{1}{c}{ Dataflow UL (bytes/s) } & \multicolumn{2}{c}{ Dataflow DL (bytes/s) } & \multicolumn{2}{c}{ Delay (s) } \\
\hline 500 & 202,340 & 185,206 & 194,794 & 191,101 & 0.124 & 0.026 \\
\hline 550 & 265,486 & 247,724 & 245,176 & 239,182 & 2.362 & 0.026 \\
\hline 600 & 311,100 & 294,019 & 191,865 & 186,745 & 5.877 & 0.022 \\
\hline 650 & 258,946 & 241,252 & 251,624 & 245,375 & 3.410 & 0.024 \\
\hline
\end{tabular}

Table 7. Results of WEB-1/P26 Scenario)

\begin{tabular}{c|c|c|c|c|c|c}
\hline \multicolumn{7}{c}{ CI-2/P26 } \\
\hline SS & \multicolumn{1}{c}{ Dataflow UL (bytes/s) } & \multicolumn{2}{c}{ Dataflow DL (bytes/s) } & \multicolumn{2}{c}{ Delay (s) } \\
\hline 500 & 204,586 & 186,773 & 136,760 & 133,426 & 0.094 & 0.022 \\
\hline 550 & 233,252 & 213,752 & 182,149 & 177,700 & 0.144 & 0.025 \\
\hline 600 & 207,639 & 188,347 & 215,503 & 210,270 & 0.124 & 0.024 \\
\hline 650 & 268,575 & 250,289 & 227,999 & 221,336 & 2.026 & 0.024 \\
\hline 700 & 284,677 & 266,132 & 233,650 & 225,346 & 3.006 & 0.023 \\
\hline 750 & 335,112 & 317,263 & 191,081 & 183,989 & 8.302 & 0.019 \\
\hline 800 & 299,195 & 281,004 & 235,513 & 226,652 & 6.005 & 0.021 \\
\hline
\end{tabular}

portant to emphasize that these numbers consider the simultaneously attending of 500 users. For only 100 users, each user will dispose of a bitrate five times the number shown in the table. For instance, for the 3.0 bandwidth, the system will offer $60 \mathrm{kbps}$, which is the same or better bitrate than that offered by dial up modems. This bitrate is appropriate for DTV interactive applications and even for Internet access. Besides, considering the overbook service typical in mobile system (with a 6 times factor), a base station with 3 sector may serve 9,000 users. These conservative numbers, by the fact that use a spectral efficiency of $2 \mathrm{bps} / \mathrm{Hz}$ and overbook factor of 6 times, show the great potential of this wireless system with high service penetration, attending the main objectives of the SBTVD-T concerning the digital divide. In fact, there is a exchange relation of frequency bandwidth and mean bitrate. The use of a channeling with bandwidth higher than 3.0 MHz, would demand more TV channels, which is not well attractive and in some areas even unfeasible. Contrarily, channeling of $1.5 \mathrm{MHz}$ may be very attractive for areas with high population density. The $2 \mathrm{MHz}$ channeling would allows a mean bitrate of $8 \mathrm{kbps}$, using four 6 $\mathrm{MHz}$ channels. These numbers show that the more attractive profiles uses $1.5 \mathrm{MHz}, 2 \mathrm{MHz}$ or $3 \mathrm{MHz}$, which correspond to use from 3 to $6 \mathrm{TV}$ channels for the return channel for the $4 \times 3$ pattern.

The numbers show in this section are only for illustrative purposes, naturally it is possible to offer large band Internet access with a higher investment on the backhaul infrastructure.
Table 9. Number of TV Channels Used and Mean Bitrate per User (500 users, TDD mode, $4 \times 3$ reuse)

\begin{tabular}{l|l|l}
\hline $\begin{array}{l}\text { Bandwidth } \\
\text { (MHz) }\end{array}$ & $\begin{array}{l}\text { Number of } \\
\text { TV Channels } \\
\text { Used }\end{array}$ & $\begin{array}{l}\text { User Bitrate } \\
\text { (kbps) } \\
\text { and downlink) }\end{array}$ \\
\hline 1.5 & 3 & 6 \\
\hline 1.75 & 4 & 7 \\
\hline 2 & 4 & 8 \\
\hline 3 & 6 & 12 \\
\hline 3.5 & 7 & 14 \\
\hline 4 & 8 & 16 \\
\hline 5.5 & 11 & 22 \\
\hline
\end{tabular}

\section{CONCLUSIONS}

The SBTVD-T is based on the modulation BSTOFDM of the ISDB-T system. Is has introduced several important improvements over the Japanese standard. The main improvements are a more flexible middleware, the use of a very efficient video codec and the return channel which is mainly focused on wireless technologies. The motivation for the search of wireless solutions is based on the observation that mobile phone use on developing countries has been increased in a very high rate at the last decades. We infer that the same rising rate will be observed in the wireless Internet access in these countries.

The Return Channel (RC) technologies defined currently in ARIB are dial-up modems, ISDN for wired lines and PDC - Personal Digital Cellular and PHS - Personal Handy-Phone System for wireless. The SBTVD- 
Table 8. Results of WEB-2/P26 Scenario)

\begin{tabular}{c|c|c|c|c|c|c}
\hline \multicolumn{7}{c}{ CI-2/P26 } \\
\hline SS & \multicolumn{7}{c}{ Dataflow UL (bytes/s) } & \multicolumn{2}{c}{ Dataflow DL (bytes/s) } & \multicolumn{2}{c}{ Delay (s) } \\
\hline 500 & 227,484 & 208,056 & 122,622 & 117,760 & 0.094 & 0.020 \\
\hline 550 & 247,398 & 226,025 & 182,011 & 176,018 & 0.129 & 0.023 \\
\hline 600 & 308,059 & 289,310 & 183,974 & 176,308 & 2.166 & 0.022 \\
\hline 650 & 286,457 & 267,596 & 205,145 & 197,910 & 1.305 & 0.024 \\
\hline 700 & 287,001 & 268,079 & 208,733 & 200,645 & 2.690 & 0.022 \\
\hline 750 & 323,344 & 305,221 & 189,734 & 180,397 & 7.215 & 0.019 \\
\hline
\end{tabular}

$\mathrm{T}$ has complemented the above return channel technologies by including WiMAX/WiMAX-700, GS/GPRS, and CDMA2000/1xRTT. This paper has reviewed some important aspects of the WiMAX-700 defined on the SBTVD-T standard.

The sector capacity obtained by the simulations is around 550 activity stations in the simulations conditions. In all combinations of traffic profiles and propagation scenarios, stationary results were obtained after a mean time delays bellow 5 seconds, for the simulations using less than 600 subscriber stations. Also the simulations have shown that the IEEE 802.16 allows simultaneously the treatment of a great number of stations. This is a very important aspect for DTV return channel, even for use in areas with high terminal density.

In the scenarios simulated in these conditions, we have obtained a net bitrate (IP layer) above $5 \mathrm{kbps}$ (uplink + downlink), this is a typical dataflow for dial-up Internet accesses. We also observed that a bitrate of up to around 8 Mbps is possible for 3.5 MHz bandwidth (IP layer). The simulated scenarios are adequate for return channels of DTV systems. For this purpose, we have considered that a user bitrate of few kilobits/s are enough for the majority of interactive applications and even for navigation on light Internet pages. The user bitrate may be much higher with the use of small cell sites, but these also imply in a higher investment on the network infrastructure.

The development of a simulation model and the simulations results have shown that the IEEE 802.16 standard presents excellent capacity and efficiency for data transmission at variable bitrates. The system allows to attain relatively high bitrates when compared to other wireless standards.

The simulations results have also shown that the system is very efficient for date bursts with high volume of data and also efficient to support a high number of terminals per sector. As far as the capacity is concerned, the system fits well for return channels in DTV, even for the high interactivity applications and it is a suitable solution for Internet access in emerging countries like Brazil.

\section{ACKNOWLEDGMENT}

The author acknowledges the support received from Finep - Financiadora de Estudos e Projetos, under grant number 01050181-00.

\section{REFERENCES}

[1] L. M. J. Barbosa; A. Budri; J. V. Gonçalves; E. Morais; R. Moreira; R. Sonntag; L. G. P. Meloni. Uma proposta para o canal de interatividade para o sbtvd através de comunicação sem fio em rf intrabanda. In XVIII Brazilian Symposium on Computer Graphics and Image Processing, pages 1-6, 2005.

[2] A. K. Budri; J. V. Gonçalves; L. G. P. Meloni. Wimax simulation models for return channel in digital television systems. In VI International Telecommunications Symposium - ITS2006, Fortaleza, 2006.

[3] Ieee standard for local and metropolitan area networks. part 16: Air interface for fixed and mobile broadband wireless access systems. amendment 2: Physical and medium access control layers for combined fixed and mobile operation in licensed bands, ieee p802.16d, 2004.

[4] Ieee standard for local and metropolitan area networks. part 16: Air interface for fixed and mobile broadband wireless access systems. amendment for physical and medium access control layers for combined fixed and mobile operation in licensed bands, ieee p802.16e, 2005.

[5] L. G. P. Meloni. Sistemas de tv digital usando antenas receptoras e transmissoras de tv para canal de retorno e como repetidor digital. Required Patent PI0304.013-5, INPI.

[6] L. G. P. Meloni. Método de canalização de sistemas de comunicação sem fio sobre o espectro de televisão e espectro adjacente. Required Patent 018060114062, INPI. 
[7] L. G. P. Meloni. Método de sincronização para receptores ofdm através da estimação dos desvios temporal e freqüencial com base na análise do sinal recebido. Required Patent 018060120940, INPI.

[8] K. Fall; K. Varadhan. The NS Manual. UC Berkeley, LBL, USC/ISI, and Xerox PARC, Apr. 2005. http://www.isi.edu/nsnam/ ns/nsdocumentation.html, Apr. 2007.

[9] C. Eklund; R. Marks; K. Stanwood; S. Wang. Ieee standard 802.16: A technical overview of the wirelessman air interface for broadband wireless access. IEEE Communications Magazine, pages 98-106, Jun. 2002.

[10] A. Ahmad; C. Xin; F. He; M. Mckormic. Multimedia performance of ieee 802.16 mac. www.cs.nsu.edu/research/OPNET/Abstracts /IEEE_802.16/ATS_2005_HF.pdf, Apr. 2007.

[11] B. Petry. 802.16 1 MAC Simulation Tools: Recommendations, Nov. 2000. http://grouper.ieee.org/ groups/802//16/tg1/mac/pres/802161mp-00_06.pdf, Apr. 2007.

[12] B. Petry. Urban transmission loss models for mobile radio in the 900 and $1800 \mathrm{mhz}$ bands, Sep. 1991.

[13] J. Cao; W.S. Cleveland; Y. Gao; K. Jeffay; F.D. Smith; M.C. Weigle. Stochastic models for generating synthetic http source traffic. In Proceedings of IEEE INFOCOM, Hong Kong, Mar. 2004.

[14] S. Ramachandran; C. W. Bostian; S. F. Midkiff. Performance evaluation of ieee 802.16 for broadband wireless acces. In Proceedings of OPNETWORK 2002, Aug. 2002.

[15] M. Pätzold. Mobile Fading Channels. John Wiley \& Sons, West Sussex, England, 2002. 